\title{
The Study of Periodic Tight Framelets and Wavelet Frame Packets and Applications
}

\author{
Shiheng Wang \\ Department of Fundamentals, Nanyang Vocational College of Agriculture, Nanyang 473000, China \\ sxxa11xauat@126.com
}

Keywords: Tight periodic wavelet frames, approximation order, periodic refinement equation, tight frame series, symmetric fames, Harmonic frames

\begin{abstract}
Information science focuses on understanding problems from the perspective of the stake holders involved and then applying information and other technologies as needed. A necessary and sufficient condition is identified in term of refinement masks for applying the unitary extension principle for periodic functions to construct tight wavelet frames. Then a theory on the approximation order of truncated tight frame series is established, which facilitates construction of tight periodic wavelet frames with desirable approximation order. The pyramid decomposition scheme is derived based on the generalized multiresolution structure.
\end{abstract}

\section{Introduction and Concepts}

The setup of tight wavelet frames provides great flexibility in approximating and representing periodic functions. Fundamentals issues involved include the construction of tight periodic wavelet frames, approximation powers of such wavelet frames, and whether wavelet frames lead to sparse representat of locally smooth periodic functions. The frame theory plays an important role in the modern time-frequency analysis. It has been developed very fast over the last twenty years, espe cially in the context of wavelets and Gabor systems. This scientific field investigates the relationship betwenrse represeen the structure of materials at atomic or molecular scales and their macroscopic properties. Wavelet theory has been applied to signal processing, image compression, and so on. Frames for a separable Hilbert space were formally defined by Duffin and Schaeffer [1] in 1952 to study some deep problems in nonharmonic Fourier series. Basically, Duffin and Schaeffer abstracted the fundamental notion of Gabor for studying signal processing [2]. These ideas did not seem to generate much general interest outside of nonharmonic Fourier series however (see Young's [3]) until the landmark paper of Daubechies, Grossmann, and Meyer [4] in 1986. After this groundbreaking work, the theory of frames began to be more widely studied both in theory and in applications [5,6], such as signal processing, image processing, data compression, sampling theory.

We begin with the unitary extension pinciple and formulate a general procedure for constructing wavelet frames. The emphasis is on having refinement masks as the starting point. The condition for this can be easily verified and also provide insight to the refinement masks that enable the construction process. Let $L^{2}[0,2 a]$ be the space of all $2 a$-periodic square-integrable complex-valued func tions over the real line $R$ with inner product $\langle\cdot, \cdot\rangle$ given by

$$
<h, v>=1 /(2 a) \int_{0}^{2 a} h(x) \overline{v(x)} d x
$$

where $h(x), v(x) \in L^{2}[0,2 a]$, and norm $\left.\|u\|_{2}=<u, u\right\rangle^{1 / 2}$. For a function $h(x) \in L^{2}[0,2 a]$, we denote its Fourier series as $\sum_{n \in Z} h(n) e^{i n}$, where $h(n)=<h, e^{i n}>, n \in Z$, are its Fourier coefficient. For any $0 \leq u \in Z$, we define the shift operator $S_{u}^{\ell}: L^{2}[0,2 a] \mapsto L^{2}[0,2 a]$ by $S_{u}^{\ell} h(x):=h\left(x-2 a \ell / 2^{u}\right)$. For $h(x) \in L^{2}[0,2 a]$, since $h(x)$ is a periodic function, it suffices to consider the shifts $S_{u}^{\ell} h(x), \ell \in \Lambda_{k}$, where $\Lambda_{k}$ is given by 


$$
\Lambda_{k}:=\left\{-2^{k-1}+1,-2^{k-1}+2, \cdots, 2^{k-1}-1,2^{k-1}\right\} .
$$

Let $\Delta\left(2^{k}\right)$ be the $2^{k}$-periodic complex sequences $b_{k}$, that is $b_{k}\left(\ell+2^{k} u\right)=b_{k}(\ell)$ for all $\ell, u \in Z$. We denote the discrete Fourier transform of $b_{k} \in \Delta\left(2^{k}\right)$ by $\hat{b}_{k}(n):=\sum_{\ell \in \Omega_{k}} b_{k}(\ell) e^{-2 a i t n / 2^{k}}$. The sequence $\hat{b}_{k}$ also lies in $\Delta\left(2^{k}\right)$. Now consider positive integers $\eta_{k}, k \geq 0$, and functions $\varphi_{0}, \psi_{k}^{r}, k \geq 0, r=1,2, \cdots, \eta_{k}$ in $L^{2}[0,2 a]$. The set $\left\{\varphi_{0}\right\} \bigcup\left\{S_{k}^{\ell} \psi_{k}^{r}, k \geq 0, r=1,2, \cdots, \eta_{k}, \ell \in \Lambda_{k}\right\}$ forms a normalized tight wavelet frame, or simply by tight wavelet frame for the space $L^{2}[0,2 a]$ if

$$
\|h\|^{2}=\left|<h, \varphi_{0}>\right|^{2}+\sum_{k=0}^{+\infty} \sum_{r=1}^{\eta_{k}} \sum_{\ell \in \Omega_{k}}\left|<h, S_{k}^{\ell} \psi_{k}^{r}>\right|^{2} .
$$

Our construction of wavelets is based a sequence of refinable functions $\left\{\varphi_{k}\right\}_{k \geq 0}$ in $L^{2}[0,2 a]$, which satisfies for every $k \geq 0$, the periodic refinement equation

$$
\varphi_{k}=\sqrt{2} \sum_{\ell \in \Omega_{k+1}} b_{k+1}(\ell) S_{k+1}^{\ell} \varphi_{k+1}
$$

for some $b_{k+1} \in \Delta\left(2^{k+1}\right)$. For each $k \geq 0$, the wavelets $\psi_{k}^{r} \in L^{2}[0,2 a], r=1,2, \cdots, \eta_{k}$ with $\eta_{k}$ be some positive integer, are given by the periodic refinement equation

$$
\psi_{k}^{r}=\sqrt{2} \sum_{\ell \in \Omega_{k+1}} d_{k+1}^{r}(\ell) S_{k+1}^{\ell} \varphi_{k+1}
$$

where $d_{k+1}^{r} \in \Delta\left(2^{k+1}\right),, r=1,2, \cdots, \eta_{k}$. Suppose that $V$ is a separable Hilbert space. We recall that a sequence $\left\{f_{k}, k \in Z\right\} \subset V$ is a frame for $V$, if there exist positive real numbers $A_{1}, A_{2}$ such that

$$
\forall \lambda \in V, \quad A_{1}\|\lambda\|^{2} \leq \sum_{v}\left|\left\langle\lambda, f_{k}\right\rangle\right|^{2} \leq A_{2}\|\lambda\|^{2} .
$$

A sequence $\left\{f_{k}, k \in Z\right\} \subset V$ is a Bessel sequence if only the upper inequaliry of (1) holds. If only for all $\left\{f_{k}, k \in Z\right\} \subset \Omega \subset V$, the upper inequality of (1) follows the sequence $\left\{f_{k}\right\} \subset V$ is a Bessel sequence with respect to (w.r.t.) $\Omega$. If $\left\{f_{k}\right\} \subset V$ is a frame there exist a dual frame $\left\{f_{k}^{*}, k \in Z\right\} \subset V$ such that

$$
\forall \xi \in \Omega, \quad \xi=\sum_{v}\left\langle\xi, f_{v}\right\rangle f_{v}^{*}=\sum_{v}\left\langle\xi, f_{v}^{*}\right\rangle f_{v}
$$

The following example gives frame-like decompositons for a subspace that are not frames. Let $\left\{f_{n}^{*}\right\}$ be a Riesz basis for a unique (biorthogonal) dual sequence $\left\{f_{n}^{0}\right\} \subset \Omega$ such that $<f_{n}^{0}, f_{k}^{*}>=\delta_{n, k}, n, k \in Z$ and

$$
\xi \in \Omega, \quad \xi=\sum_{v \in Z}\left\langle\xi, f_{v}^{*}\right\rangle f_{v}^{0}=\sum_{v \in Z}\left\langle\xi, f_{v}^{0}\right\rangle f_{v}^{*}
$$

In the context of biorthogonal bases within $V$,this is usually the end of it. However, choose now a function $\Delta f_{n} \in \Omega^{\perp} \subset V$, and let $f_{n}=f_{n}^{0}+\Delta f_{n}$. It obviously follows that

$$
\left\langle f_{n}, f_{k}^{*}\right\rangle=\left\langle f_{n}^{0}+\Delta f_{n}, f_{k}^{*}\right\rangle=\left\langle f_{n}^{0}, f_{k}^{*}\right\rangle+0=\delta_{n, k}
$$

and for all $\xi \in \Omega, \quad \sum_{v \in Z}\left\langle\xi, f_{v}\right\rangle f_{v}^{0}=\sum_{v \in Z}\left\langle\xi, f_{v}^{0}+\Delta f_{v}\right\rangle f_{v}^{*}=\xi$. The conclusion is that there are infinitely many biorthogonal duals $\left\{f_{k}\right\}$ to a biorthogonal basis $\left\{f_{k}^{*}\right\}$ of subspace $\Omega$, so long if $\left\{f_{k}\right\}$ is allowed to go beyond $\Omega$. 


\section{Multiple Pseudoframe and Generalized Multiresolution Structure}

Let $J=\{1,2, \cdots s\}$ be a finite index set where $s$ be a positive constant integer. We consider the case of multiple generators, which yield multiple pseudoframes for subspaces of $L^{2}(R)$.

Definition 1. Let $\left\{T_{k} \Upsilon_{j}\right\}$ and $\left\{T_{k} \widetilde{\Upsilon}_{j}\right\} \quad(j \in J, k \in Z)$ be two sequences in $V$, We say $\left\{T_{k} \Upsilon_{j}\right\}$ forms a multiple pseudoframe for $\Omega \subset V$ with respect to (w.r.t.) $\left\{T_{k} \widetilde{\Upsilon}_{j}\right\}$, if

$$
\forall f(x) \in \Omega, \quad f(x)=\sum_{j \in J} \sum_{k \in Z}\left\langle f, T_{k} \Upsilon_{j}\right\rangle T_{k} \widetilde{\Upsilon}_{j}
$$

where we define a translate operator, $T_{k} \Upsilon(x)=\Upsilon(x-k)$, for a function $\Upsilon(t) \in L^{2}(R)$.

Definition 2. A Generalized multiresolution structure (GMS) $\left\{U_{k}, f_{j}, \widetilde{f}_{j}\right\}$ is a sequence of closed linear subspaces $\left\{U_{k}\right\}$ of $L^{2}(R)$ and $2 r$ elements $f_{j}, \tilde{f}_{j} \in L^{2}(R) \quad(j \in J)$ such that (a) $U_{k} \subset U_{k+1}$; (b) $\bigcap_{k \in Z} U_{k}=\varnothing, \bigcup_{k \in Z} U_{k}=L^{2}(R)$; (c) $\hbar(x) \in U_{k}$ if and only if $D \hbar(x) \in U_{k+1}$, where the dilation operator $D f(x)=2^{1 / 2} f(2 x)$, for $f(x) \in L^{2}(R)$; (d) $\hbar(x) \in U_{0}$ implies $\hbar(x-k) \in U_{0}$, for all $k \in Z$; (e) $\left\{T_{k} f_{j}\right\}$ forms a multiple pseudoframes for $U_{0}$ with respect to $\left\{T_{k} \widetilde{f}_{j}\right\}$ $(j \in J, k \in Z)$.

\section{Construction of Affine Pseudoframes for $\operatorname{Space} L^{2}(R)$}

In order to split a function $f(x)$ of $U_{1}$ into two functions (mostly) in $S_{0}$ and $W_{0}$, respectively, we will construct an affine pseudoframe for $U_{0}$ making use of the existing affine pseudoframe structure for $U_{0}$. Conventional symbols, $\Upsilon_{l}(x)$ and $\widetilde{\Upsilon}_{l}(x)$, will be used as generating functions for $W_{0}$. But they need notbe contained in $W_{0}$.

Definition 3. Let $\left\{U_{k}, f(x), \widetilde{f}(x)\right\}$ be a given GMS, and let $\Upsilon_{l}(x)$ and $\widetilde{\Upsilon}_{l}(x) \quad(l \in J)$ be 2s band-passfunctions in $L^{2}(R)$. We say $\left\{T_{v} f(x), T_{v} \widetilde{\Upsilon}_{l}(x), l \in J\right\}$ forms an affine pseudoframe for $U_{1}$ with respect to $\left\{T_{v} \widetilde{f}(x), T_{v} \widetilde{\Upsilon}_{l}(x), l \in J\right\}$, if

$$
\forall \Phi(x) \in U_{1}, \Phi(x)=\sum_{v \in Z}\left\langle\Phi, T_{v} \tilde{f}\right\rangle T_{v} f(x)+\sum_{l \in J} \sum_{v \in Z}\left\langle\Phi, T_{v} \widetilde{\Upsilon}_{l}\right\rangle T_{v} \Upsilon_{l}(x),
$$

Accordingly, $\left\{T_{v a} \tilde{f}, T_{v a} \widetilde{\Upsilon}_{l}, \imath \in J\right\}$ is called a dual affine pseudoframes with respect to $\left\{T_{v} f, T_{v} \Upsilon_{\imath}\right\} \quad(\imath \in J)$ in the sense of (6).

Then there exists a bivariate function $f(x) \in L^{2}(R)$ (see ref.[3]) such that

$$
f(x)=\sqrt{2} \sum_{v \in Z} d_{0}(v) f(2 x-v) .
$$

There exists a scaling relationship for function $\widetilde{f}(x)$ under the same conditions as that of $d_{0}$ for a sequence $\widetilde{d_{0}}$, that is

$$
\widetilde{f}(x)=\sqrt{2} \sum_{s \in Z} \widetilde{d_{0}}(s) \widetilde{f}(2 x-s) .
$$

To characterize the condition for which $\left\{T_{v} f, T_{v} \Upsilon_{l}, l \in J\right\}$ forms an affine pseudoframe for 
$V_{1}$ with respect to $\left\{T_{v} \widetilde{f}, T_{v} \widetilde{\Upsilon}_{l}, l \in J\right\}$, we begin with developing the wavelet refinement equations associated with band-pass functions $\Upsilon_{l}(l \in J)$ and $\widetilde{\Upsilon}_{l}(l \in J)$ based on a generalized multiresolution structure, namely,

$$
\begin{aligned}
& \Upsilon_{l}(x)=\sqrt{2} \sum_{v \in Z} m_{l}(v) f(2 x-v), \quad l \in J \quad \text { in } L^{2}(R), \\
& \widetilde{\Upsilon}_{l}(x)=\sqrt{2} \sum_{v \in Z} \widetilde{m}_{l}(v) \widetilde{f}(2 x-v), \quad l \in J \quad \text { in } L^{2}(R) .
\end{aligned}
$$

Let $\chi_{\Lambda}(\omega)$ be the characteristic function of the interval $\Lambda$ defined in Proposition 1 . We shall use the following 1-periodic function. $\Gamma_{\Lambda}(\omega) \equiv \sum_{k} \chi_{\Lambda}(\omega+k)$ Theorem 2. Let $\Lambda$ be the bandwidth of the subspace $V_{0}$ defined in Proposition 1. $\left\{T_{v} f, T_{v} \Upsilon_{l}, v \in Z, l \in J\right\}$ forms an affine pseudoframe for $V_{1}$ with respect to $\left\{T_{v} \widetilde{f}, T_{v} \widetilde{\Upsilon}_{l}, l \in J\right\}$ if and only if there exist $D_{0}$ and $D_{l},(l \in J)$ in $\left.L^{2}([0,1])\right)$ such that

$$
\begin{gathered}
\sum_{l \in J} D_{l}(\omega) \overline{\widetilde{M}_{l}(\omega)} \Gamma_{\Lambda}(\omega)=2 \Gamma_{\Lambda}(\omega) \\
\sum_{l \in J} D_{l}(\omega+1 / 2) \overline{\widetilde{M}_{l}(\omega+1 / 2)} \Gamma_{\Lambda}(\omega)=0
\end{gathered}
$$

Theorem 1. Let $f(x), \widetilde{f}(x), \Upsilon_{t}(x)$ and $\widetilde{\Upsilon}_{l}(x), l \in J$ be functions in $L^{2}(R)$ defined by (7), (8), (9) and (10) respectively. Assume that conditions in Definition 1 are satisfied. Then, for any function $\Psi(x) \in L^{2}(R)$, and any integer $\mathrm{n}$,

$$
\sum_{k \in Z}\left\langle\Psi, \widetilde{f}_{n, k}\right\rangle f_{n, k}(x)=\sum_{i=1}^{s} \sum_{v=-\infty}^{n-1} \sum_{k \in Z}\left\langle\Psi, \widetilde{\Upsilon}_{l: v, k}\right\rangle \Upsilon_{t: v, k}(x) .
$$

Moreover, for any univariate function $\Psi(x) \in L^{2}(R), \quad \Psi(x)=\sum_{l=1}^{s} \sum_{v=-\infty}^{+\infty} \sum_{k \in Z}\left\langle\Psi, \widetilde{\Upsilon}_{l: v, k}\right\rangle \Upsilon_{t: v, k}(x)$. Consequently, if $\left\{\Upsilon_{t: v, k}(x)\right\}$ and $\left\{\widetilde{\Upsilon}_{t: v, k}(x)\right\}, \quad(i \in J, v \in Z, \quad k \in Z)$ are also Bessel sequences, they are a pair of affine frames for the space $L^{2}(R)$.

\section{Conclusion}

The multiple affine pseudoframes for the subspaces of $L^{2}(R)$ are studied. The pyramid decomposition scheme is derived based on such a GMS. As a major new con stribution the constructionof affine frames for $L^{2}(R)$ based on a GMS is presented.

\section{Acknowledgment}

This work was supported by the Science Research Foundation of Education Department of Shaanxi Provincial Government (Grant No: 2013JK0564).

\section{References}

[1] S. Li, M. Ogawa: Pseudoframes for Subspaces with Applications. J. Fourier Anal. Appl. Vol 10, pp409-431.(2004).

[2] R. J. Duffin, A. C. Schaeffer: A class of nonharmonic Fourier series , Trans. Amer. Math. Soc., Vol. 72, pp. 341-366.(1952). 
[3] S. Li: A Theory of Geeneralized Multiresolution Structure and Pseudoframes of Translates. J. Fourier Anal. Appl. Vol 6(1), pp 23-40. (2001).

[4] A.Ron, Z. Shen: Affine systems in $L^{2}\left(R^{d}\right)$. (II) Dual systems. J. Fourier Anal. Appl. Vol 4, pp617-637.( 1997).

[5] Q. Chen, et al. The characterization of a class of subspace pseudoframes with arbitrary real num -ber translations. Chaos, Solitons \& Fractals, 2009,42:2696-2706.

[6] Q. Chen, A. Huo. The research of a class of biorthogonal compactly supported vector-valued wavelets. Chaos, Solitons \& Fractals, 2009,41(2): 951-961. 